OPEN ACCESS

Edited by:

Yong Xia,

Jining Medical University, China

Reviewed by:

Hong Jiang,

Sichuan University, China

Hong Liu,

Central South University, China

*Correspondence:

Yang Xu

xuyang2020@zju.edu.cn

Lili Yu

2321128@zju.edu.cn

${ }^{t}$ These authors have contributed equally to this work

Specialty section:

This article was submitted to Gastrointestinal and Hepatic Pharmacology,

a section of the journal

Frontiers in Pharmacology

Received: 09 January 2022 Accepted: 25 January 2022

Published: 02 March 2022

Citation:

Jiang $T$, Yang J, Yang $H$, Chen W, JiK, $X U Y$ and $Y u L$ (2022) SLC35B4 Stabilizes C-MYC Protein by OGlcNAcylation in HCC. Front. Pharmacol. 13:851089. doi: 10.3389/fphar.2022.851089

\section{SLC35B4 Stabilizes c-MYC Protein by O-GICNAcylation in HCC}

\author{
Tao Jiang ${ }^{1 \dagger}$, Jinghong Yang ${ }^{1 \dagger}$, Huohong Yang ${ }^{1}$, Wancheng Chen ${ }^{1}$, Kaiyuan $\mathrm{Ji}^{1}$, Yang $\mathrm{Xu}^{1,2 *}$ \\ and Lili $Y^{3,4 *}$ \\ ${ }^{1}$ School of Basic Medical Sciences, Southern Medical University, Guangzhou, China, ${ }^{2}$ Department of Cardiology, Cardiovascular \\ Key Lab of Zhejiang Province, The Second Affiliated Hospital, Zhejiang University School of Medicine, Zhejiang University, \\ Hangzhou, China, ${ }^{3}$ Department of Medical Oncology, Key Laboratory of Cancer Prevention and Intervention, Ministry of \\ Education, The Second Affiliated Hospital, Zhejiang University School of Medicine, Hangzhou, China, ${ }^{4}$ Cancer Center, Zhejiang \\ University, Hangzhou, China
}

UDP-GlcNAc is a sugar substrate necessary for the O-GlcNAcylation of proteins. SLC35B4 is one of the nucleotide sugar transporters that transport UDP-GlcNAc and UDP-xylose into the endoplasmic reticulum and Golgi apparatus for glycosylation. The roles of SLC35B4 in hepatocellular carcinoma (HCC) tumorigenesis remain unknown. We find that the expression levels of SLC35B4 are higher in HCC tissues than adjacent nontumor tissues. SLC35B4 is important for the proliferation and tumorigenesis of HCC cells. Mechanistically, SLC35B4 is important for the O-GlcNAc modification of c-Myc and thus the stabilization of C-Myc, which is required for HCC tumorigenesis. Therefore, SLC35B4 is a promising therapeutic target for treating HCC.

Keywords: O-GIcNAcylation, HCC, c-Myc, SLC35B4, nucleotide sugar transporters

\section{INTRODUCTION}

Liver cancer is one of the most common human cancers and the third leading cause of cancer death in 2020 (Sung et al., 2021). Hepatocellular carcinoma (HCC) is the major type of primary liver cancer (Sung et al., 2021). The main risk factors causing HCC include chronic infection with hepatitis B virus or hepatitis $\mathrm{C}$ virus, heavy alcohol use, and non-alcoholic fatty liver disease usually associated with obesity and type 2 diabetes (Rawla et al., 2018). Available therapeutic options for HCC include tumor resection, liver transplantation, percutaneous ethanol injection, and radiofrequency ablation (Karaman et al., 2014; Llovet et al., 2021). However, the effective therapies for advanced HCC are limited due to the lack of understanding of the pathways driving HCC (Jeng et al., 2015). Therefore, to develop more effective HCC therapy, it is important to identify new therapeutic targets that drive HCC tumorigenesis.

O-linked $\mathrm{N}$-acetylglucosaminylation (O-GlcNAcylation) is a common post-translational modification of the serine or threonine residues of protein, which occurs in the endoplasmic reticulum (ER) and Golgi apparatus using UDP-N-acetylglucosamine (UDP-GlcNAc) as the substrate (Hart et al., 2011). This modification is catalyzed by O-GlcNAc transferase (OGT) and removed by O-GlcNAcase (OGA) (Ong et al., 2018). Previous studies have shown that O-GlcNAcylation, the new hallmark of cancer, promotes tumorigenesis via multiple mechanisms, including the regulation of cell cycle, chromatin dynamics, and tumor metastasis (Fardini et al., 2013). Elevated O-GlcNAcylation enhances glycolysis by regulating the activity of key glycolytic enzymes, including GLUT1, PFK1, and PGK1 (Bacigalupa et al., 2018; Nie et al., 2020). O-GlcNAcylation has been shown to regulate the stability of c-MYC and HIF-1a via direct or indirect manners, which are two oncogenic transcriptional factors critical for tumor progression 
(Itkonen et al., 2013; Ferrer et al., 2014). Nucleotide sugar transporters (NSTs) are a family of transport proteins that move the glycosylation substrates across the ER or Golgi apparatus membranes (Handford et al., 2006; Hadley et al., 2014). Solute carrier family 35 member B4 (SLC35B4) is one member of NSTs that can transport both UDP-GlcNAc and UDP-xylose (Ashikov et al., 2005). A previous study has demonstrated that SLC35B4 is regulated by oncoprotein YAP1 and promotes gastric cancer development and progression (Liu et al., 2019). However, the roles of SLC35B4 in promoting tumorigenesis remain unknown.

In this study, we showed that the expression levels of SLC35B4 in HCC are higher than normal liver tissues, and the overexpression of SLC35B4 is correlated with the poor prognosis of cancer patients. SLC35B4 knockdown dramatically decreased the proliferation and migration of HCC cells. Mechanistically, while SLC35B4 knockdown did not affect the mRNA levels of c-Myc, it significantly decreased c-Myc protein levels. In addition, we discovered that SLC35B4 knockdown in HCC cells decreased the O-GlcNAcylation of c-MYC that is known to stabilize c-Myc. Therefore, SLC35B4 drives HCC progression by stabilizing c-Myc through O-GlcNAc modification of c-Myc.

\section{MATERIALS AND METHODS}

\section{Cell Lines and Cell Culture}

Hepatocellular carcinoma cell line (HepG2) was obtained from ATCC. Hepatocellular carcinoma cell line (QGY-7703) was provided by the Pathology Department of Sun Yat-sen University Cancer Center. HEK 293FT was purchased from Thermo Fisher Scientific. All of the cell lines were cultured in Dulbecco's Modified Eagle Medium (DMEM) containing 10\% fetal bovine serum (FBS) and $1 \%$ penicillin/streptomycin at $37^{\circ} \mathrm{C}$ with $5 \% \mathrm{CO}_{2}$.

\section{Animals and Human HCC Samples}

All animal experiments were performed according to the protocols approved by the Institutional Animal Care and Use Committee (IACUC) of Southern Medical University. For xenograft tumor growth, $5 \times 10^{6}$ cells were injected subcutaneously into the left (control group) and the right (SLC35B4 knockdown group) flanks of the immunodeficient NSG mice (purchased from Shanghai Model Organisms, Shanghai, China. $n=6$ ), respectively. For inducible gene knockdown, the drinking water containing $2 \mathrm{mg} / \mathrm{L}$ of
A
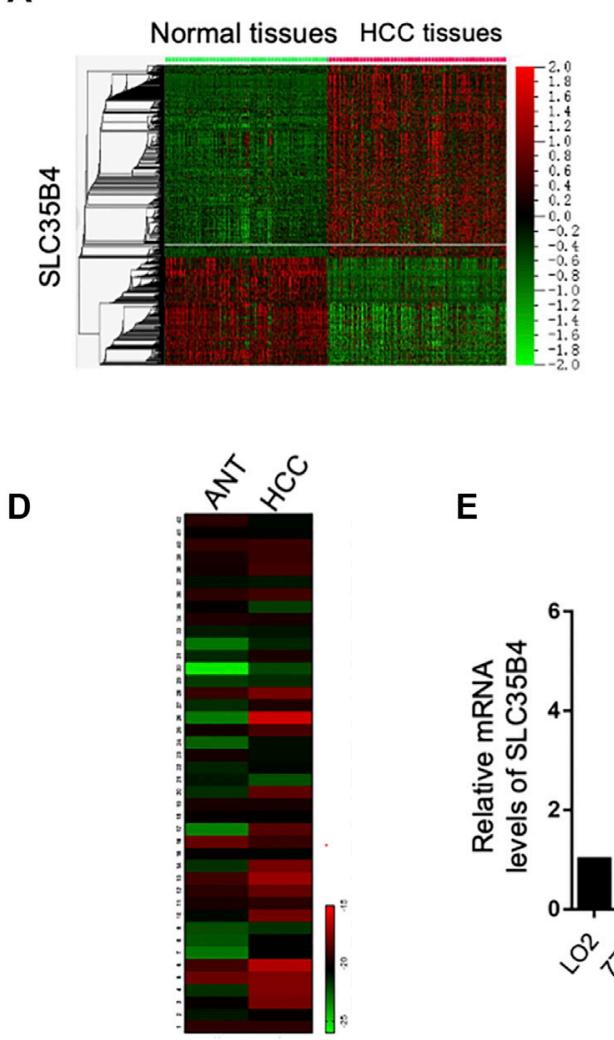

B

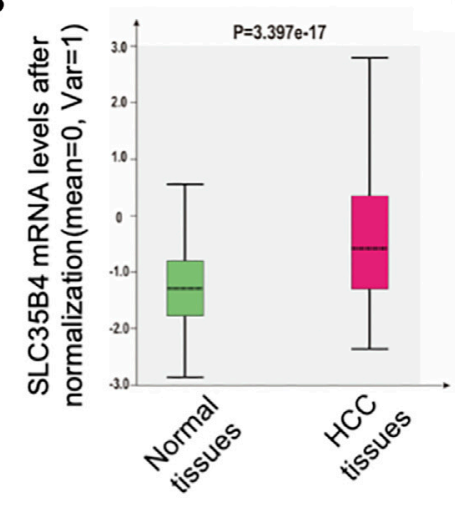

C

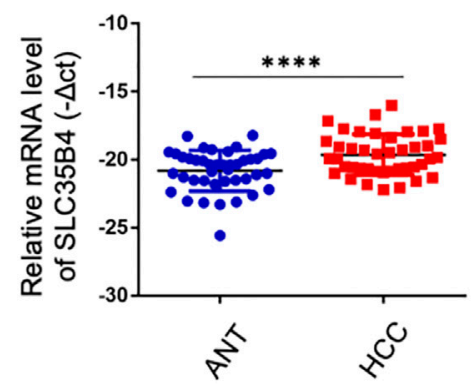

E

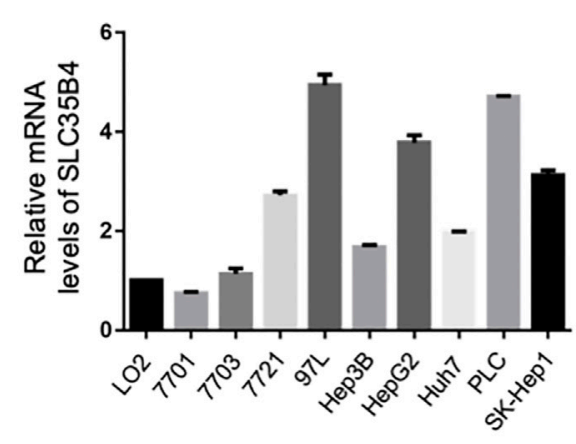

$\mathbf{F}$

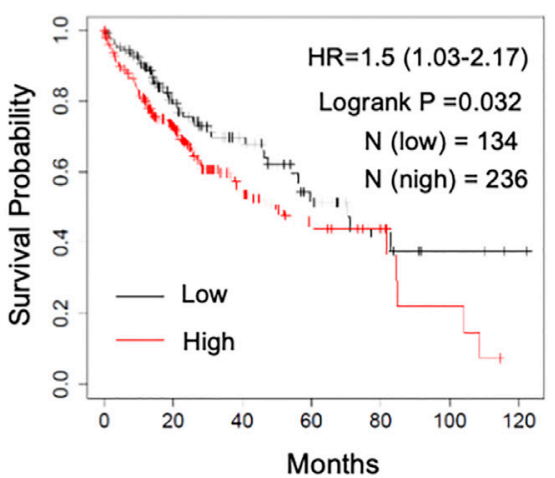

FIGURE 1 | SLC35B4 is overexpressed in HCC and inversely correlated with prognosis of HCC patients. (A) The heat map of mRNA expression profile in 268 nontumor adjacent tissues and 243 HCC tissues in GSE25097 dataset. The mRNA expression level of SLC35B4 was indicated by a white line. (B) The box plot of SLC35B4 expression levels in non-tumor tissues (268) and HCC tissues (243) in GSE25097 dataset. $P$-value is indicated. (C,D) The relative mRNA expression levels of SLC35B4 in 42 paired adjacent normal tissues (ANT) and HCC tissues. P-value is indicated. (E) The mRNA expression levels of SLC35B4 in HCC cell lines. N=3. Data are presented as mean \pm SD. (F) Kaplan-Meier survival curve of the overall survival of HCC patients with high and low SLC35B4 expression levels. The SLC35B4 expression levels were inversely correlated with the overall survival of HCC patients. $P$-value is indicated. 
doxycycline was supplied with doxycycline $(20 \mathrm{mg} / \mathrm{kg}$ body weight) injected intraperitoneally every day.

After obtaining adequate informed consent, HCC tissue and adjacent normal tissue (ANT, exceeding the edge of the tumor by at least $2 \mathrm{~cm}$ ) were obtained from HCC patients who underwent curative resection for HCCs in Nanfang Hospital of Southern Medical University, Guangzhou, China, between November 2010 and May 2015. This study was approved by IRB of Nanfang Hospital at Southern Medical University and was performed according to the Declaration of Helsinki (6th revision, 2008).

\section{Data Analysis From TCGA}

GSE25097 dataset analysis was obtained from Gene Expression Omnibus (GEO) database (https://www.ncbi.nlm.nih.gov/geo/ query/acc.cgi?acc=GSE25097). Kaplan-Meier survival curves of overall survival in HCC patients were plotted according to the data from a previous study (Nagy et al., 2021).

\section{Cellular Proliferation and Clonal Formation Assays}

HCC cells were digested by $0.25 \%$ trypsin at $37^{\circ} \mathrm{C}$ for $3 \mathrm{~min}$ and washed with PBS. For cellular proliferation assay, cells $(2,000$ cells/well or 5,000 cells/well) were seeded into a 96-well plate. Twenty-four hours later, $10 \mu \mathrm{l}$ of CCK8 solution was added into each well and incubated at $37^{\circ} \mathrm{C}$ for $1 \mathrm{~h}$ before the absorbance was detected at $450 \mathrm{~nm}$ using a microplate reader. Every experiment at least had three repetitions. For clonal formation assay, cells (500 cells/well) were seeded into a 6-well plate and incubated at $37^{\circ} \mathrm{C}$ for about 2 weeks. After fixation by $100 \%$ methanol at room temperature for $15 \mathrm{~min}$, the cell colonies were stained with $0.1 \%$ crystal violet for $1 \mathrm{~h}$ at room temperature and counted. Every experiment at least had three repetitions.

\section{Cell Lysis and Western Blotting Analysis}

After washing with PBS and harvested, HCC cells were sonicated at $4^{\circ} \mathrm{C}$ in RIPA buffer (50 mM Tris- $\mathrm{HCl} \mathrm{pH} \mathrm{8.0,150} \mathrm{mM} \mathrm{NaCl,}$ $1 \%$ Triton X-100, $0.5 \%$ sodium deoxycholate, $0.1 \%$ SDS) containing $1 \%$ protease inhibitor cocktail (Thermo Fisher Science). Lysate suspension was obtained after centrifugation at $4^{\circ} \mathrm{C}$ for $10 \mathrm{~min}$ and the protein concentration determined using BCA assay kit (Sigma). Protein denaturation was performed at $100^{\circ} \mathrm{C}$ for $5 \mathrm{~min}$ in $1 \times$ sample buffer (Bio-Rad). For Western blotting, the same amount of protein was loaded onto PAGE gel and transferred to a PVDF membrane (Merck) by tank transfer system (Bio-Rad). After being blocked by $5 \%$ milk at room temperature for $1 \mathrm{~h}$, primary antibody was incubated with the membrane at $4^{\circ} \mathrm{C}$ overnight. Immunoblot signal was detected using ChemiDoc Touch Imaging System (Bio-Rad) after the incubation with secondary antibody at room temperature for $1 \mathrm{~h}$.

\section{Real-Time PCR}

Total RNA from cells was extracted using RNeasy Mini Kit (Invitrogen) according to the manufacturer's instruction. Briefly, cells were lysed in RLT lysis buffer and homogenized using a 1-ml syringe with needle. RNA was purified with RNeasy Mini Kit columns and finally dissolved in RNase-free water. cDNA synthesis from total RNA was performed using PrimeScript RT reagent Kit (Takara) following the manufacturer's protocol. TB Green Premix Ex Taq II (Takara) was used for Real-Time PCR detection according to the manufacturer's instruction. Every experiment at least had three repetitions.

\section{Construct and Lentivirus Production}

For SLC35B4 knockdown, two different SLC35B4 shRNA target sequences were synthesized and inserted into pLKO.1-puro vector (Addgene 8453) and tet-pLKO-puro vector (Addgene 21915). For lentivirus production, package plasmid psPAX2 (Addgene 12260), envelop plasmid pMD2.G (Addgene 12259), and the pLKO.1 vectors were co-transfected into HEK 293FT cells. Forty-eight hours after transfection, the supernatant was harvested and concentrated with Lenti-X concentrator (Clontech). The lentivirus was stored at $-80^{\circ} \mathrm{C}$.

\section{Cell Migration Assay}

For cell migration assay, culture media $(500 \mu \mathrm{l})$ containing $10 \%$ FBS was added into the wells of a 24 -well plate with $1 \times 10^{5}$ cells cultured in the inside compartment of a Transwell insert supplemented with DMEM media without FBS. Twenty hours after incubation, the cells attached to the membrane of the Transwell insert were fixed and stained by $0.1 \%$ crystal violet. Every experiment at least had three repetitions.

\section{Immunoprecipitation Assay}

HCC cells were harvested and were lysed using Pierce IP lysis buffer (25 mM Tris- $\mathrm{HCl} \mathrm{pH} 7.4,150 \mathrm{mM} \mathrm{NaCl}, 1 \mathrm{mM}$ EDTA, $1 \%$ NP-40, and 5\% glycerol). Protein concentration was determined by BCA assay. For immunoprecipitation assay, $1 \mathrm{mg}$ of total lysate was incubated with anti-c-Myc or anti-OGlcNAc antibody $4^{\circ} \mathrm{C}$ overnight. After the incubation with Protein $A$ and $G$ magnetic beads at room temperature for $1 \mathrm{~h}$, the beads were collected and washed 3 to 5 times using lysis buffer. The beads were heated at $100^{\circ} \mathrm{C}$ for 5 min with $2 \times$ sample buffer and stored at $-80^{\circ} \mathrm{C}$.

\section{Statistical Analysis}

The statistical significance of Kaplan-Meier survival plot was determined by log-rank analysis. The other statistical significance was detected by $t$-test. All of the statistical analyses were performed in GraphPad Prism. ${ }^{\star} p<0.05,{ }^{* *} p<0.01$, ${ }^{* * *} p<$ 0.001 , and ${ }^{* * *} p<0.0001$; n.s. means non-significant.

\section{RESULTS}

\section{SLC35B4 is Overexpressed in HCC and Correlated With the Poor Prognosis of HCC Patients}

To examine the potential involvement of SLC35B4 in HCC, the GSE25097 dataset of HCC patients was analyzed, indicating that the SLC35B4 gene was significantly higher in HCC than in adjacent normal tissues (Figures $\mathbf{1 A}, \mathbf{B}$ ). In addition, the analysis of SLC35B4 mRNA levels in 42 paired HCC tissues 


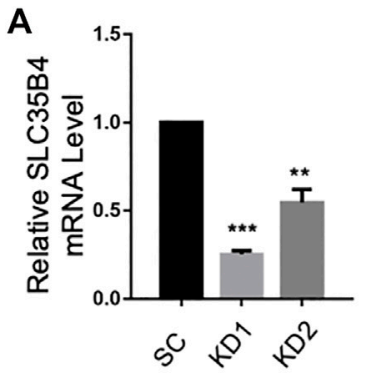

E

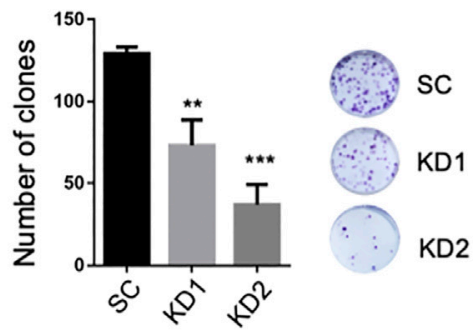

H

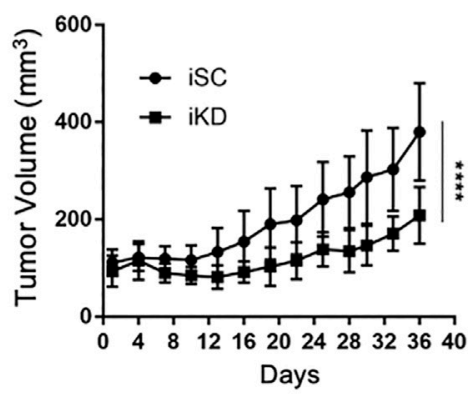

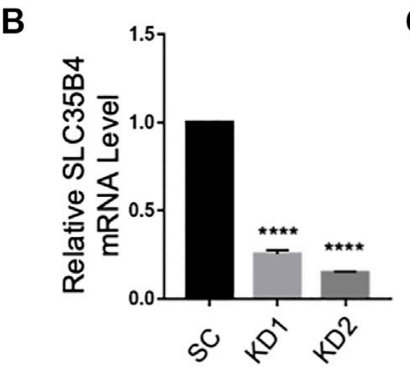

$\mathbf{F}$
C

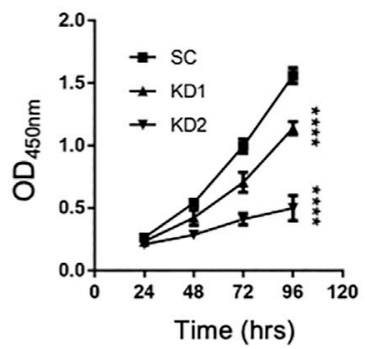

D

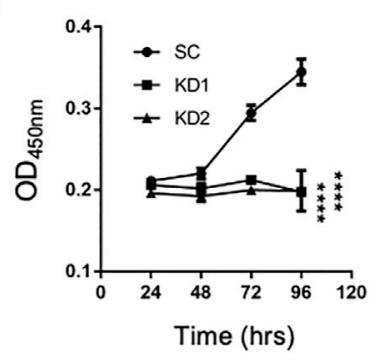

G
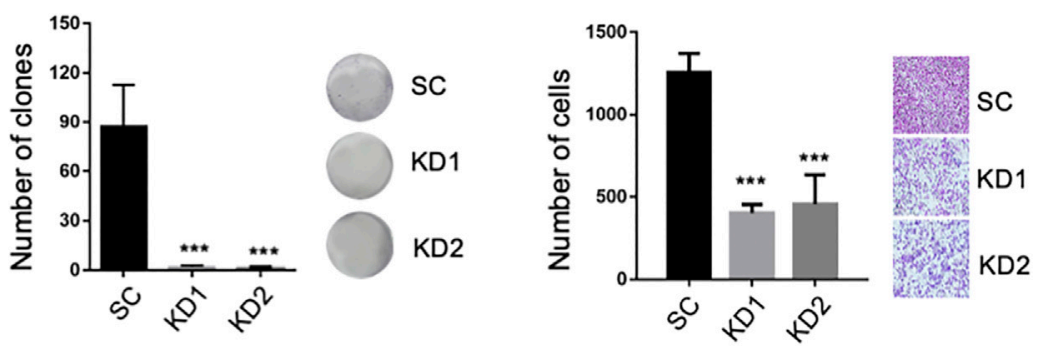

I
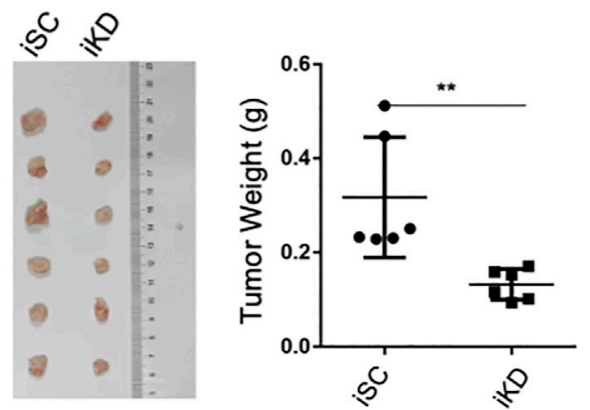

FIGURE 2 | SLC35B4 promotes HCC cell proliferation and migration. (A,B) Relative mRNA expression levels of SLC35B4 in HCC cells after SLC35B4 knockdown. $N=3$. Data are presented as mean \pm SD. (C,D) SLC35B4 knockdown significantly decreased the cellular proliferation of HCC cells as determined by CCK8. $N=3$. Data are presented as mean \pm SD. (E,F) SLC35B4 knockdown dramatically decreased the clonal formation ability of $7703(\mathbf{E})$ and HepG2 (F) cells. $N=3$. Data are presented as mean \pm SD. (G) SLC35B4 knockdown inhibited the migration of HCC cells in vitro. $N=3$. Data are presented as mean \pm SD. (H) The volume of xenograft tumors after the inducible knockdown of SLC35B4. iSC, inducible scrambled control; iKD, inducible knockdown. (I) The image and weight of xenograft tumors formed by HCC cells with or without inducible SLC35B4 knockdown in NSG mice 37 days after inducible knockdown. $N=6$. Data are presented as mean $\pm S D$. ${ }^{*} p<0.05,{ }^{* *} p<0.01$, ${ }^{\star \star *} p<0.001,{ }^{\star \star \star \star} p<0.0001$.

and adjacent non-tumor tissues further confirmed this conclusion (Figures 1C,D). SLC35B4 was also widely expressed in HCC cell lines, including HepG2, PLC, and 7703 cells (Figure 1E). The overexpression of SLC35B4 is correlated with the poor prognosis of the HCC patients (Figure 1F). These findings suggest that SLC35B4 plays important roles in driving HCC development.

\section{SLC35B4 Promotes the Proliferation and Migration of HCC Cells}

To study the roles of SLC35B4 in HCC progression, we knocked down SLC35B4 in HCC cell lines 7703 and HepG2 using two specific short hairpin RNAs (Figures 2A,B). The results showed that SLC35B4 knockdown significantly suppressed the proliferation and colony formation capability of HCC cells (Figures 2C-F). In addition, SLC35B4 knockdown suppressed the migration of HCC cells (Figure 2G). Consistent with these findings, the inducible knockdown of SLC35B4 dramatically suppressed tumor growth in the immunodeficient NODSCID mice (Figures 2H,I). These results demonstrate that SLC35B4 is important for HCC tumorigenesis in vitro and in vivo.

\section{SLC35B4 Stabilizes c-Myc via O-GIcNAc Modification}

c-Myc is a critical oncogenic transcription factor that directly binds to the promoters of oncogenes and plays key roles in driving cancer progression (Dang, 2012). O-GlcNAc 
A

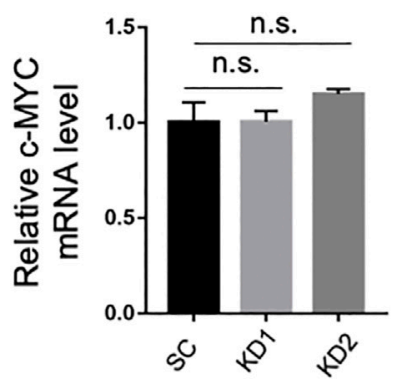

B

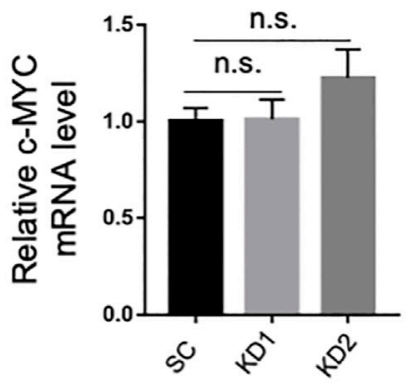

E

CHX: $0 \begin{array}{lllllllll}0 & 0.2 & 0.5 & 1 & 0 & 0.2 & 0.5 & 1 & \mathrm{hr}\end{array}$

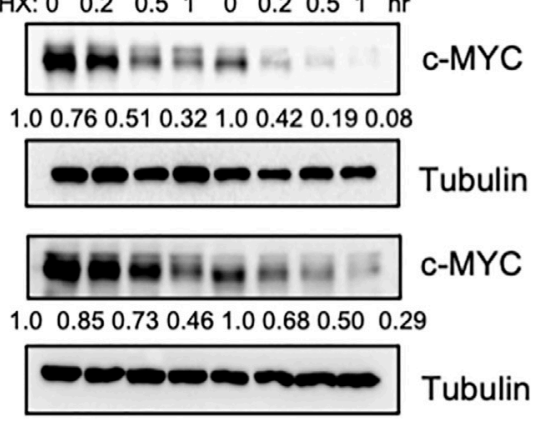

G

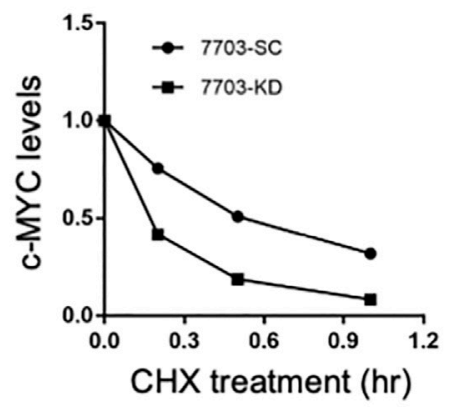

C

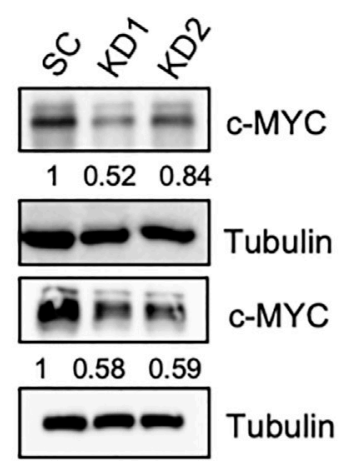

F

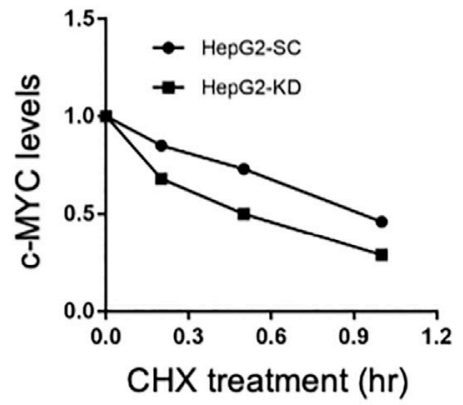

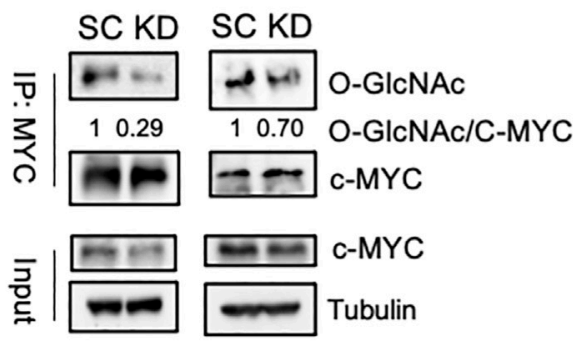

FIGURE 3 |SLC35B4 stabilizes c-Myc via O-GlcNAc modification. (A,B) Relative mRNA expression levels of c-Myc in 7703 (A) and HepG2 (B) cells with SLC35B4 knockdown. $N=3$. Data are presented as mean \pm SD. n.s., non-significant. (C) The protein levels of c-Myc in 7703 (upper) and HepG2 (bottom) cells with SLC35B4 knockdown. The relative protein levels are indicated. (D) The protein levels of c-Myc protein in 7703 (upper) and HepG2 (bottom) cells after CHX treatment. (E,F) Quantification of the protein levels of c-Myc in 7703 (E) and HepG2 (F) cells with or without SLC35B4 knockdown at different time points after CHX treatment. The relative protein levels are indicated. (G) The O-GlcNAcylation of c-Myc after SLC35B4 knockdown. c-Myc in 7703 (left) and HepG2 (right) cells with or without SLC35B4 knockdown was immunoprecipitated and the levels of the O-GlcNAcylation were detected with anti-O-GlcNAc antibody. The relative levels of O-GlcNAcylated versus total c-Myc are indicated.

modification of c-Myc protein by O-linked $\beta$-N-acetylglucosamine transferase (OGT) can stabilize c-Myc protein in cancer cells (Itkonen et al., 2013). Considering the involvement of SLC35B4 in glycosylation, we speculated that SLC35B4 could regulate the expression of c-Myc. Therefore, we examined the mRNA and protein levels of c-Myc in HCC cells before and after SLC35B4 knockdown. While SLC35B4 KD had no impact on the mRNA levels of c-Myc, it significantly reduced c-Myc protein levels by destabilizing c-Myc (Figures 3A-C). Mechanistically, we showed that SLC35B4 knockdown dramatically decreased the protein stability and the O-GlcNAc modification of c-Myc (Figures 3D-G). Considering the important roles of $\mathrm{c}-\mathrm{Myc}$ in driving the turnorigenesis of NPC, these results demonstrate that SLC35B4 drives HCC progression by stabilizing c-Myc through O-GlcNAcylation.

\section{DISCUSSION}

HCC remains one of the most lethal malignancies that lack effective therapy. To develop effective therapies and new therapeutics to treat HCC, extensive international effort has been devoted to identify new pathways that drive HCC tumorigenesis. In this study, we provide compelling evidence 
that SLC35B4, one member of the nucleotide sugar transporters required to transport nucleotide sugars into the ER or Golgi apparatus for protein glycosylation (Bazan et al., 2018), is overexpressed in HCC and drives HCC tumorigenesis. In this context, the knockdown of SLC35B4 inhibits the proliferation and migration of HCC cells in vitro, and the acute depletion of SLC35B4 in HCC tumors significantly suppresses the tumor growth in vivo. Therefore, SLC35B4 represents a promising new therapeutic target to treat HCC.

To understand the mechanism underlying SLC35B4dependent tumorigenesis, based on the physiological functions of SLC35B4 in transporting substrates of O-GlcNAc modification into the Golgi and ER, we investigated oncogenic proteins that are regulated by O-GlcNAc modification. Our study demonstrates that SLC35B4 is important to stabilize c-Myc by promoting its $\mathrm{O}-\mathrm{GlcNAc}$ modification.

$\mathrm{c}-\mathrm{Myc}$ is a critical oncoprotein that is overexpressed in many types of human cancer and plays key roles in driving tumorigenesis (Pelengaris et al., 2002). Therefore, c-Myc could be an ideal therapeutic target to treat various types of cancers. However, due to the critical roles of c-Myc in normal cellular processes, the complete inactivation of $\mathrm{c}-\mathrm{Myc}$ will have lethal effects on normal cells (Carabet et al., 2018). Therefore, c-Myc remains an undruggable target in cancer drug discovery. The destabilization of c-Myc protein in human cancers has become a promising strategy to target $\mathrm{c}-\mathrm{Myc}$. Therefore, while it remains to be confirmed, our findings that SLC35B4 depletion could destabilize c-Myc provide an alternative approach to target $\mathrm{c}-\mathrm{Myc}$ in many types of human cancer.

\section{REFERENCES}

Ashikov, A., Routier, F., Fuhlrott, J., Helmus, Y., Wild, M., Gerardy-Schahn, R., et al. (2005). The Human Solute Carrier Gene SLC35B4 Encodes a Bifunctional Nucleotide Sugar Transporter with Specificity for UDP-Xylose and UDP-NAcetylglucosamine. J. Biol. Chem. 280, 27230-27235. doi:10.1074/jbc. M504783200

Bacigalupa, Z. A., Bhadiadra, C. H., and Reginato, M. J. (2018). O-GlcNAcylation: Key Regulator of Glycolytic Pathways. J. Bioenerg. Biomembr 50, 189-198. doi:10.1007/s10863-018-9742-3

Bazan, B., Wiktor, M., Maszczak-Seneczko, D., Olczak, T., Kaczmarek, B., and Olczak, M. (2018). Lysine at Position 329 within a C-Terminal Dilysine Motif Is Crucial for the ER Localization of Human SLC35B4. PLoS One 13, e0207521. doi:10.1371/journal.pone.0207521

Carabet, L. A., Rennie, P. S., and Cherkasov, A. (2018). Therapeutic Inhibition of Myc in Cancer. Structural Bases and Computer-Aided Drug Discovery Approaches. Int. J. Mol. Sci. 20, 120. doi:10.3390/ijms20010120

Dang, C. V. (2012). MYC on the Path to Cancer. Cell 149, 22-35. doi:10.1016/j.cell. 2012.03.003

Fardini, Y., Dehennaut, V., Lefebvre, T., and Issad, T. (2013). O-GlcNAcylation: A New Cancer Hallmark? Front. Endocrinol. (Lausanne) 4, 99. doi:10.3389/fendo. 2013.00099

Ferrer, C. M., Lynch, T. P., Sodi, V. L., Falcone, J. N., Schwab, L. P., Peacock, D. L., et al. (2014). O-GlcNAcylation Regulates Cancer Metabolism and Survival Stress Signaling via Regulation of the HIF-1 Pathway. Mol. Cel 54, 820-831. doi:10.1016/j.molcel.2014.04.026

Hadley, B., Maggioni, A., Ashikov, A., Day, C. J., Haselhorst, T., and Tiralongo, J. (2014). Structure and Function of Nucleotide Sugar Transporters: Current Progress. Comput. Struct. Biotechnol. J. 10, 23-32. doi:10.1016/j.csbj.2014. 05.003

\section{DATA AVAILABILITY STATEMENT}

The original contributions presented in the study are included in the article/Supplementary Material, further inquiries can be directed to the corresponding authors.

\section{ETHICS STATEMENT}

The animal study was reviewed and approved by IACUC, Southern Medical University.

\section{AUTHOR CONTRIBUTIONS}

Conceptualization: LY and YX. Methodology: TJ, LY, and YX. Investigation: TJ, JY, HY, WC, and KJ. Formal analysis: TJ, LY, and YX. Resources: LY and YX. Writing-original draft: TJ, LY, and YX. Writing-review and editing: all authors. Supervision: LY and YX. Project administration: LY and YX. Funding acquisition: LY and $Y X$.

\section{FUNDING}

This study was supported by the National Natural Science Foundation of China (Nos. 81930084 and 81902506), and the Key Research and Development Program of Guangdong Province (2019B020235003).

Handford, M., Rodriguez-Furlán, C., and Orellana, A. (2006). Nucleotide-sugar Transporters: Structure, Function and Roles In Vivo. Braz. J. Med. Biol. Res. 39, 1149-1158. doi:10.1590/s0100-879x2006000900002

Hart, G. W., Slawson, C., Ramirez-Correa, G., and Lagerlof, O. (2011). Cross Talk between O-GlcNAcylation and Phosphorylation: Roles in Signaling, Transcription, and Chronic Disease. Annu. Rev. Biochem. 80, 825-858. doi:10.1146/annurev-biochem-060608-102511

Itkonen, H. M., Minner, S., Guldvik, I. J., Sandmann, M. J., Tsourlakis, M. C., Berge, V., et al. (2013). O-GlcNAc Transferase Integrates Metabolic Pathways to Regulate the Stability of C-MYC in Human Prostate Cancer Cells. Cancer Res. 73, 5277-5287. doi:10.1158/0008-5472.CAN-13-0549

Jeng, K. S., Chang, C. F., Jeng, W. J., Sheen, I. S., and Jeng, C. J. (2015). Heterogeneity of Hepatocellular Carcinoma Contributes to Cancer Progression. Crit. Rev. Oncol. Hematol. 94, 337-347. doi:10.1016/j. critrevonc.2015.01.009

Karaman, B., Battal, B., Sari, S., and Verim, S. (2014). Hepatocellular Carcinoma Review: Current Treatment, and Evidence-Based Medicine. World J. Gastroenterol. 20, 18059-18060. doi:10.3748/wjg.v20.i47.18059

Liu, J., Zhao, X., Wang, K., Zhang, X., Yu, Y., Lv, Y., et al. (2019). A Novel YAP1/ SLC35B4 Regulatory axis Contributes to Proliferation and Progression of Gastric Carcinoma. Cell Death Dis 10, 452. doi:10.1038/s41419-019-1674-2

Llovet, J. M., Kelley, R. K., Villanueva, A., Singal, A. G., Pikarsky, E., Roayaie, S., et al. (2021). Hepatocellular Carcinoma. Nat. Rev. Dis. Primers 7, 6. doi:10.1038/ s41572-020-00240-3

Nagy, Á., Munkácsy, G., and Győrffy, B. (2021). Pancancer Survival Analysis of Cancer Hallmark Genes. Sci. Rep. 11, 6047. doi:10.1038/s41598-021-84787-5

Nie, H., Ju, H., Fan, J., Shi, X., Cheng, Y., Cang, X., et al. (2020). O-GlcNAcylation of PGK1 Coordinates Glycolysis and TCA Cycle to Promote Tumor Growth. Nat. Commun. 11, 36. doi:10.1038/s41467-019-13601-8

Ong, Q., Han, W., and Yang, X. (2018). O-GlcNAc as an Integrator of Signaling Pathways. Front. Endocrinol. (Lausanne) 9, 599. doi:10.3389/fendo.2018.00599 
Pelengaris, S., Khan, M., and Evan, G. (2002). c-MYC: More Than Just a Matter of Life and Death. Nat. Rev. Cancer 2, 764-776. doi:10.1038/nrc904

Rawla, P., Sunkara, T., Muralidharan, P., and Raj, J. P. (2018). Update in Global Trends and Aetiology of Hepatocellular Carcinoma. Contemp. Oncol. (Pozn) 22, 141-150. doi:10.5114/wo.2018.78941

Sung, H., Ferlay, J., Siegel, R. L., Laversanne, M., Soerjomataram, I., Jemal, A., et al. (2021). Global Cancer Statistics 2020: GLOBOCAN Estimates of Incidence and Mortality Worldwide for 36 Cancers in 185 Countries. CA Cancer J. Clin. 71, 209-249. doi:10.3322/caac. 21660

Conflict of Interest: The authors declare that the research was conducted in the absence of any commercial or financial relationships that could be construed as a potential conflict of interest.
Publisher's Note: All claims expressed in this article are solely those of the authors and do not necessarily represent those of their affiliated organizations, or those of the publisher, the editors, and the reviewers. Any product that may be evaluated in this article, or claim that may be made by its manufacturer, is not guaranteed or endorsed by the publisher.

Copyright $\odot 2022$ Jiang, Yang, Yang, Chen, Ji, Xu and Yu. This is an open-access article distributed under the terms of the Creative Commons Attribution License (CC $B Y)$. The use, distribution or reproduction in other forums is permitted, provided the original author(s) and the copyright owner(s) are credited and that the original publication in this journal is cited, in accordance with accepted academic practice. No use, distribution or reproduction is permitted which does not comply with these terms. 